\title{
Digit Ratio (2D:4D) and cancer : What is known so far?
}

\author{
Renato Nicolás Hopp ${ }^{1}$, Nathalia Caroline Souza Lima ${ }^{1}$, José Laurentino Ferreira Filho', \\ Marcondes Sena-Filho', Renata Oliveira Samuel' ${ }^{2}$, Jackeline Gallo Amaral' ${ }^{2}$, Jacks Jorge ${ }^{1}$ \\ ${ }^{I}$ Piracicaba Dental School, Universidade Estadual de Campinas, Piracicaba, Brazil. \\ ${ }^{2}$ Aracatuba Dental School, Universidade Estadual Paulista, Aracatuba, Brazil.
}

Received January 14, 2014; Revised January 27, 2014; Accepted January 28, 2014; Published Online January 30, 2014

\section{Review Article}

\begin{abstract}
The ratio between the second and fourth digits is a proxy marker for prenatal exposure and sensitivity to sexual hormones, which can be genetically influenced. The influence of prenatal hormone exposure can reflect on adult life traits such as psychological traits, athletic performance and diseases such as cardiovascular. An important and newly explored field on digit ratio research is its correlation to different types of cancer, as a marker for prevalence and severity. In this review, the different types of cancer already correlated to digit ratios are discussed.
\end{abstract}

Keywords: Prenatal testosterone; Digit ratio; Cancer

\section{Introduction}

The ratio between the second and fourth digits, also known as digit ratio or $2 \mathrm{D}: 4 \mathrm{D}$ is a proxy marker for the prenatal influence of sexual hormones - mainly testosterone and estrogen. It is negatively correlated to prenatal testosterone (PT), i.e., the lower the ratio between the index and ring finger, the higher the exposure and sensitivity to PT. Conversely it is positively correlated to prenatal estrogen (PE). There is evidence that prenatal exposure and sensitivity to testosterone are inversely correlated to $2 \mathrm{D}: 4 \mathrm{D}$, especially in the right hand, as well as to the difference between the right and left 2D:4D $\left(\Delta_{\mathrm{r}-1}\right){ }^{1,2}$

The exposure and sensitivity to PT and PE is regulated genetically. Nineteen genes have been identified to influence in digit ratio establishment in mice ${ }^{3}$ and the HOX, androgen receptor (AR) and LIN28B have been speculated to reflect on digit ratio in humans ${ }^{4,5}$ Each gene is either correlated to PT or $\mathrm{PE}$, being up or down regulated by the exposure to these hormones, but no gene is influenced in the same direction by PT and PE. This feature makes digit ratio a candidate marker for the action of these genes, and, subsequently for it use as a

Corresponding author: Renato Nicolás Hopp; Piracicaba Dental School, Universidade Estadual de Campinas, Piracicaba, Brazil. Email: renhopp@gmail.com

Cite this article as:

Hopp RN, Lima N, Filho J, Sena-Filho M, Samuel RO, Amaral JG, Jorge J. Digit Ratio (2D:4D) and cancer: What is known so far? Int $J$ Cancer Ther Oncol 2014; 2(1):020111.

DOI: $10.14319 /$ ijcto.0201.11 predictor of disease susceptibility.

In recent years, three main work fronts have studied 2D:4D psychological traits, body performance and adult-life disease susceptibility. As evidence was set out that 2D:4D may be predictive of susceptibility to cancers and that this may be particularly true in cancers that show sex differences in their occurrence, progression, and/or prognosis ${ }^{6}$, studies started to focus on the relationship between PT/PE and the susceptibility to prostate, breast and gastric cancers, among others. This review will focus on the studies investigating the correlations between 2D:4D and different types of cancer based on studies retrieved from PubMed, Scopus and Google Scholar using the keywords 'digit ratio', '2D:4D' and 'cancer'.

\section{Prostate cancer}

Prostate cancer is the cancer most connected to $2 \mathrm{D}: 4 \mathrm{D}$, which is being studied by several groups across the globe. However, not all studies can be compared due to differences in methodology and the choice of evaluation of one or both hands. Groups of different countries have evaluated the influence of right-hand 2D:4D, left-hand 2D:4D, as well as $\Delta_{\mathrm{r}-1}$ over the prevalence and severity of prostate cancer, as well as prostatic-specific antigen and Gleason scores.

The first study to evaluate the correlations between 2D:4D and prostate cancer was performed in Korea. ${ }^{7}$ This study did not find a relationship between prostate volume and 2D:4D, but encountered significant negative correlation between 
2D:4D and PSA, in a sample of 366 men. The researchers evaluated only the right hand and, after dividing the patients into low $(<0.95)$ and high $(>0.95)$ digit ratio, found significant higher risks for prostate biopsy and prostate cancer $(\mathrm{OR}=1.75$ and 3.22, respectively). Another Korean study found an association between digit ratio, prostate cancer volume and Gleason score, after investigating 770 men with lower urinary tract symptoms. ${ }^{8}$ Again, the patients were divided into low or high digit ratio. The "low" group presented higher cancer detection rates $(\mathrm{p}<0.005)$ and more voluminous tumors $(\mathrm{p}=0.005)$ as well as higher Gleason scores $(\mathrm{p}=$ 0.01).

A larger sample was analyzed in a cohort study in the United Kingdom, based on data from 4568 patients. ${ }^{9}$ This study found negative correlations between 2D:4D and prostate cancer and suggested protective effects of prenatal testosterone $(\mathrm{OR}=0.67)$, especially in younger patients. This study, however, analyzed hand patterns of the right hand with self-reports of patients of high or low ratio between the index and ring fingers. It must be noted that sometimes the looks of the ratio between the index and ring fingers can be deceitful. Nonetheless, given the large sample size, possible discrepancies on the analysis tend to be diminished.

A smaller but much admixed population was analyzed in Brazil for prostate cancer prevalence, and compared to a sample of benign prostatic hyperplasia (BPH). ${ }^{10}$ Once again, only right-hands were evaluated and males with prostate cancer presented lower digit ratios than the ones with no prostatic lesions. The authors suggested 2D:4D as a possible screening marker for prostate cancer incidence. A rather larger sample studied different ethnic background patients, comparing Caucasians to African-Americans in the USA. ${ }^{11}$ This study found lower right-hand digit ratios for African-Americans with prostate cancer in comparison to Caucasians ( $\mathrm{p}<0.001$ ), but no significant differences for Gleason scores or age at diagnosis, among other features.

A Spanish group tried to evaluate digit ratio as a predictor for prostate cancer in a sample of 204 men $^{12}$ using left-hand measurements correlated to PSA, sexual-hormone binding protein and Gleason scores. This study found a positive correlation between high digit ratios and prostate cancer incidence $(\mathrm{OR}=4.4)$, especially in older patients. Conversely, The only study to evaluate both hands digit ratio and its correlations to prostate cancer - performed in Australia with a large sample of 6258 men (686 cases) ${ }^{13}$ - found no correlation between right or left 2D:4D after age 60, but found an inverse association for younger prostate cancer patients, inferring that 2D:4D could be a marker for cancer screening of early onset prostate cancer.

Regarding prostate cancer, the many studies published in the past years still do not point in the same direction. The dif- ferent results may reside in the different methodologies (choice of hand of evaluation, technique use, data analysis) or in the different ethnic backgrounds of the samples (largely admixed or not, comparisons between Caucasians and African-Americans). As prostate cancer is a multifactorial disease, influenced by race and dietary habits, among others, the role of PT can be important, and larger multi-center studies may be necessary to clarify the possible role of digit ratios on the screening of this disease.

\section{Testicular cancer}

A single study has investigated the correlations between 2D:4D and testicular cancer ${ }^{14}$, evaluating 71 cases and 122 controls. This study found no correlations between testicular cancer and right-hand digit ratios. Other traits such as weight at birth and testicular volume were negatively correlated to $2 \mathrm{D}: 4 \mathrm{D}$ by the same study, pointing to other factors that can influence testicular cancer.

\section{Female cancers}

Little has been published so far with regard to the correlations between female cancer and digit ratio. One study has investigated the correlation between 2D:4D, human papillomavirus (HPV) and cervical dysplasia in a British sample ${ }^{15}$ evaluating both right and left hands and found significantly higher digit ratios for HPV-positive women compared to HPV-negative. There was also a similar trend for persistent HPV infection. Moreover, women with cervical dysplasia had significantly higher digit ratios than HPV-negative women, regardless of ethnic background. The study concluded that PT has a negative correlation with persistent HPV infections and risk of cervical intraepithelial neoplasia

Recently, the first study on digit ratio and breast cancer has been published by an Australian group. ${ }^{2}$ This study analyzed 573 breast cancer patients in a universe of 9044 women, investigating right, left and $\Delta_{\mathrm{r}-1} 2 \mathrm{D}: 4 \mathrm{D}$ and found a positive correlation between left hand 2D:4D and breast cancer risk, but negative correlations for $\Delta_{\mathrm{r}-\mathrm{l}}$. Right-hand digit ratios along with $\Delta_{\mathrm{r}-1}$ were negatively correlated with age at diagnosis, possibly being correlated to early onset breast cancer, especially $\Delta_{\mathrm{r}-1}$.

\section{Oral cancer}

One study has investigated the role of PT on the prevalence of oral squamous cell carcinoma, the most common mouth cancer. ${ }^{16}$ The investigators compared the right hand digit ratios of oral cancer patients, oral premalignant lesions patients and healthy subjects, matched by age and tobacco consumption. Oral cancer patients presented higher right-hand 2D:4D than subjects with oral premalignant lesions and healthy individuals $(\mathrm{p}<0.05)$, pointing to an influence of the lack of prenatal androgen exposure on the onset of mouth cancer. Further studies regarding other types or oral cancer (salivary gland tumors, lip cancer) would be 
welcome in the literature, as well as a comparison of tobacco-using habits (chewing, smoking and inverted smoking).

\section{Gastric cancer}

A recent study has correlated gastric cancer to $2 \mathrm{D}: 4 \mathrm{D}^{17}$, in a pattern similar to the breast cancer study by Muller et. $a l^{2}$. Overall, 116 individuals, age and sex matched were compared (57 cases and 59 controls). Left-hand 2D:4D (positively) and $\Delta_{\mathrm{r}-1}$ (negatively) were associated to gastric cancer, particularly for males $(\mathrm{p}=0.001$ and $\mathrm{p}=0.0003$ respectively). The authors also investigated the correlations between 2D:4D and cancer staging, but found no significant correlation.

\section{Discussion and Conclusion}

Prenatal testosterone and prenatal estrogen influence the formation and establishment of digit ratios ${ }^{3}$ and can also influence the onset of psychological, behavioral or medical traits. ${ }^{6,19}$ Digit ratio was proposed as a possible marker for disease predisposition in $2000^{19}$ and as a possible marker for the action of genes shortly thereafter. ${ }^{4,18}$

Today, it is known that 2D:4D can be a proxy marker for the action of about 22 genes, 3, 4, 5, 18 many of which are connected to different types of cancer. Moreover, each gene is influenced either positively or negatively by PT or PE, and never in the same direction, both in women or men

The relationship between 2D:4D and cancer was first hypothesized for breast cancer, ${ }^{18,20}$ but the first study to confirm a correlation was published in 2012. ${ }^{2}$ Before that, correlations between 2D:4D and cancer had been established for prostate and mouth tumors, as well as for cervical cancer risk.

As the influence of PT/PE - reflected on the ratio between the $2^{\text {nd }}$ and $4^{\text {th }}$ digits seems to be especially true for sex-influenced cancers, there is still a broad field for investigations of the correlations between digit ratio and cancers. Even for the cancers already correlated to digit ratio, the exact directions in which $\mathrm{PT} / \mathrm{PE}$ influence carcinogenesis is yet to be established. Further studies are welcome in the literature regarding digit ratio and cancer.

\section{Conflict of interest}

The authors declare that they have no conflicts of interest. The authors alone are responsible for the content and writing of the paper.

\section{References}

1. Breedlove SM. Minireview: Organizational hypothesis: instances of the fingerpost. Endocrinology 2010; 151:4116-22.

2. Muller DC, Baglietto L, Manning JT, McLean C, Hopper JL, English DR, Giles GG, Severi G. Second to fourth digit ratio (2D:4D), breast cancer risk factors, and breast cancer risk: a prospective cohort study. Br JCancer 2012; 107:1631-6.

3. Zheng Z, Cohn MJ. Developmental basis of sexually dimorphic digit ratios. Proc Natl Acad Sci US A 2011; 108:16289-94.

4. Manning JT, Callow M, Bundred PE. Finger and toe ratios in humans and mice: implications for the aetiology of diseases influenced by HOX genes. Med Hypotheses 2003; 60:340-3.

5. Medland SE, Zayats T, Glaser B, Nyholt DR, Gordon SD, Wright MJ, Montgomery GW et al. A variant in LIN28B is associated with 2D:4D finger-length ratio, a putative retrospective biomarker of prenatal testosterone exposure. Am J Hum Genet 2010; 86:519-25.

6. Manning JT. Digit Ratio: A Pointer to Fertility, Behavior, and Health. Rutgers University Press; 2002.

7. Jung H, Kim KH, Yoon SJ, Kim TB. Second to fourth digit ratio: a predictor of prostate-specific antigen level and the presence of prostate cancer. BJU Int 2011; 107:591-6.

8. Oh JK, Kim KH, Jung H, Yoon SJ, Kim TB. Second to fourth digit ratio: its relationship with core cancer volume and Gleason score in prostate biopsy. Int Braz J Urol 2012; 38:611-9.

9. Rahman AA, Lophatananon A, Stewart-Brown S, Harriss D, Anderson J, Parker T et al. Hand pattern indicates prostate cancer risk. Br J Cancer 2011; 104:175-7.

10. Hopp RN, Jorge J. Right hand digit ratio (2D:4D) is associated with prostate cancer: Findings of an admixed population study. Journal of Solid Tumors 2012; 2: 22-25.

11. Waters M, Rebholz CM, Wood B, Kuske A, McIntyre M, Sartor O. Second to fourth digit ratio and prostate cancer severity. Prostate Cancer Prostatic Dis 2013; 16:107-10.

12. Garcia-Cruz E, Piqueras $M$, Huguet J, Ribal MJ, Vilaseca A, Gosalbez D et al. Higher second fourth digit ratio predicts higher incidence of prostate cancer in prostate biopsy. Arch Esp Urol 2012; 65:816-21.

13. Muller DC, Giles GG, Manning JT, Hopper JL, English DR, Severi G. Second to fourth digit ratio (2D:4D) and prostate cancer risk in the Melbourne Collaborative Cohort Study. Br J Cancer 2011; 105:438-40. 
14. Auger J, Eustache F. Second to fourth digit ratios, male genital development and reproductive health: a clinical study among fertile men and testis cancer patients. Int J Androl 2011; 34:e49-58.

15. Brabin L, Roberts SA, Farzaneh F, Fairbrother E, Kitchener HC. The second to fourth digit ratio (2D:4D) in women with and without human papillomavirus and cervical dysplasia. Am J Hum Biol 2008; 20:337-41.

16. Nicolas Hopp R, Jorge J. Right hand digit ratio (2D:4D) is associated with oral cancer. Am J Hum Biol 2011; 23:423-5.
17. Nicolás Hopp R, de Souza Lima NC, Filho JL, Filho MS, Lima CS, Jorge J. Digit ratio (2D:4D) is associated with gastric cancer. Early Hum Dev 2013; 89:327-9.

18. Manning JT, Bundred PE, Newton DJ, Flanagan BF. The second to fourth digit ratio and variation in the androgen receptor gene. Evol Hum Behav 2003; 24: 399-405.

19. Manning JT, Bundred PE. The ratio of 2nd to 4 th digit length: a new predictor of disease predisposition? Med Hypotheses 2000; 54:855-7.

20. Trichopoulos D. Hypothesis: does breast cancer originate in utero? Lancet 1990; 335:939-40. 